\title{
Mechanism of tiron as scavenger of superoxide ions and free electrons
}

\author{
Fatai A. Taiwo \\ Leicester School of Pharmacy, De Montfort University, Leicester, LE1 9BH, UK
}

\begin{abstract}
Sodium 4,5-dihydroxybenzene-1,3-disulfonate (tiron) has been reported to be an efficient chelator of certain metal ions, and a substrate in several enzyme reactions. Its small size facilitates cell entry and therefore modulates intracellular electron transfer reactions as an antioxidant by scavenging free radicals. Its reduction by electrochemical and enzymatic methods gives identical products; a semiquinone detectable by EPR spectroscopy. In a test of its use as a spin trap, in comparison with DMPO, tiron does not form a molecular spin-adduct but proves more functional as an electron trap. Electron addition to tiron is more facile than reduction of dioxygen as observed by the non-formation of DMPO-OOH spin-adduct in the system $\mathrm{XO} / \mathrm{HPX} / \mathrm{O}_{2} / \mathrm{DMPO} /$ tiron. Rather, it is the tiron semiquinone radical which is formed quantitatively with increasing concentration of hypoxanthine independent of oxygen concentration. These results offer explanation for the action of tiron and its suitability for measuring electron release in hypoxic conditions, and also for mitigating redox-induced toxicity in drug regimes by acting as an electron scavenger.
\end{abstract}

Keywords: Tiron, free radicals, spin trap, electrons, EPR

\section{Introduction}

Reactive oxygen species, superoxide and hydroxyl radicals in particular have been implicated in many pathogenic conditions. The removal of these radicals and metal ions capable of Fenton reaction is the major function of many endogenous antioxidants in biochemical systems. Sodium 4,5-dihydroxybenzene1,3-disulfonate (tiron) (I) has proved a multipurpose reagent in this respect. Tiron is used as metal ion chelating agent for the determination and detoxification of trace amounts of metal ions [1,2], as a superoxide radical scavenger [3], preferential cell-permeable radical scavenger [4,5], and substrate in electron transfer reactions [6]. Apart from metal chelation where there is mere substitution of cations preferably with higher binding constants, other reactivities involve formation of redox intermediates, principally the tiron semiquinone which is detectable by electron paramagnetic resonance (EPR) spectroscopy.

In its mode of action, tiron has been described as a spin trap and the semiquinone product as a radical adduct. Whereas in conventional spin-trapping formalism the substrate reacts with the radical to form specific molecular adducts identifiable by their characteristic EPR parameters, tiron forms no such adducts but the semiquinone radical (Scheme 1) [7].

The radical scavenging reaction mechanism of tiron is thought to be its direct oxidation by first-formed superoxide radical species:

$$
\begin{aligned}
& \mathrm{TH}_{2}+\mathrm{O}_{2}^{--}+\mathrm{H}^{+} \rightarrow \mathrm{TH}^{\cdot}+\mathrm{H}_{2} \mathrm{O}_{2}, \\
& \mathrm{TH}^{\cdot} \rightarrow \mathrm{T}^{--}+\mathrm{H}^{+},
\end{aligned}
$$

to generate the tiron semiquinone and peroxide [8]. In anaerobic conditions, where there is no superoxide 
<smiles>O=S(=O)(O)c1cc(O)c(O)c(S(=O)(=O)O[Na])c1</smiles>

Tiron (I)

Scheme 1.

radical generated, tiron would be susceptible to direct attack by free electrons. Though direct reduction of tiron has not been reported, its reactivity with hydrated electrons or radiolytic and electrolytic products of water are clear possibilities.

In an early publication, tiron had been reported to protect cells against aerobic toxicity of tirapazamine (SR4233), a proposed anticancer drug [6]. In a more recent publication we demonstrated the formation of radicals from the bioreduction of tirapazamine [9]. These radicals, we concluded, were the cytotoxic species derived from the pro-drug and essential for disrupting cellular processes in-hypoxia. These two reports would appear to indicate the possibility of differential protection of aerobic cells by tiron during hypoxic cytotoxicity of tirapazamine - a conjoint therapy. This notion calls to question the uniqueness of tiron in mitigating free radical or electron-mediated reactions or in redox reactions especially in oxic media.

This study examines the primary reactivities of tiron with the superoxide radical anion, and the hydrated electron. A comparison with the spin trap 5,5-dimethyl-1-pyrroline-N-oxide (DMPO) is made to differentiate between electron trapping and conventional spin trapping in free radical reactions. The many functions of tiron including enzyme inhibition calls to question its characteristic role either as a radical trapping agent or an electron trap in biological systems.

\section{Materials and methods}

Sodium 4,5-dihydroxybenzene-1,3-disulfonate (tiron), 5,5-dimethyl-1-pyrrolidine-N-oxide (DMPO), xanthine oxidase (type 1), and hypoxanthine were obtained from Sigma Chemical Co. DMPO was purified by double filtration through activated charcoal and syringe filter Whatman $0.2 \mu \mathrm{m}$ pore size with a PTFE filter medium. Concentration of DMPO solution was routinely determined using a molar extinction coefficient of $\varepsilon_{234}=7700 \mathrm{M}^{-1} \mathrm{~cm}^{-1}$ [10]. Typical concentration of hypoxanthine used was $0.9 \mathrm{mM}$ with $0.08 \mathrm{U}$ of xanthine oxidase. Concentration of DMPO was $100 \mathrm{mM}$.

Experimental solutions were made usually in two parts; one containing $10 \mathrm{mM}$ tiron in PBS pH 7.4 and $0.08 \mathrm{U}$ xanthine oxidase, the other containing $100 \mathrm{mM}$ DMPO with $0.09 \mathrm{mM}$ hypoxanthine. Tiron and DMPO were omitted at different times as required but the total volume of final solution was maintained at $350 \mu$ l.

Solutions were deoxygenated by exhaustive purging with oxygen-free nitrogen in a glove box in which all subsequent procedures were performed. Solutions were exposed to air for oxygenation by ambient oxygen. Higher levels of oxygenation were achieved by flushing with oxygen gas. Oxygen content was routinely monitored with a Clarke oxygen electrode (Rank Brothers, Cambridge, England) calibrated with a solution of sodium dithionite for 'zero' and oxygen saturated PBS for $240 \mu \mathrm{M} \mathrm{O}_{2}$ [11].

For EPR measurements a quartz flat cell previously modified by sealing to its lower end, two fine cannulas such that syringes could be used for simultaneous injection of solutions. The ends of both 
cannulas were adjusted to meet at the same point a short distance below the flat section of the cell. This device had proved efficient for the simultaneous delivery of two solutions for in-situ generation and trapping of radicals in-cavity [12], one containing the enzyme xanthine oxidase, and the other the substrate hypoxanthine. Cavity tuning was generally accomplished and spectrum acquisition commenced within $55 \mathrm{~s}$ of injection. All EPR measurement were made on a Bruker 6/1 EMX spectrometer operating at X-band frequency range $(9.7 \mathrm{GHz})$ set at $100 \mathrm{kHz}$ modulation. $g$-Values were obtained by the internal Brucker autoscaler and calculated with reference to 2,2-diphenyl-1-picrylhydrazyl hydrate (DPPH) free spin $\left(g_{\mathrm{e}}=2.00232\right)$ field marker. Instrument settings were as follows: field set $348.9 \mathrm{mT}$, sweep width $3.0 \mathrm{mT}$, microwave power $20 \mathrm{~mW}$, time constant $10.24 \mathrm{~ms}$, sweep time $83 \mathrm{~s}$. EPR kinetic scans were obtained with microwave current $10 \mathrm{~mW}$, field set $349.4 \mathrm{mT}$, sweep width $0 \mathrm{mT}$, modulation amplitude $0.05 \mathrm{mT}$, time constant $20.48 \mathrm{~s}$ and sweep time $2684 \mathrm{~s}$.

In-cavity electrolysis was performed by the application of $5 \mathrm{~mA}$ current from a Bruker current source (ER164 EC) to platinum electrodes inserted at the top and bottom of the aqueous flat cell. For electrolysis, only the solution of tiron was filled into the flat cell.

EPR spectra were recorded as first derivative plots of intensity versus Hall field. Simulation of spectra was performed using SimFonia program (Bruker Spectrospin).

\section{Results and discussion}

Detection of the superoxide radical anion, formed in the xanthine oxidase-catalysed oxidation of xanthine, by EPR spectroscopy was facilitated by spin trapping using DMPO. The DMPO-OOH adduct has a characteristic 12-line spectrum; $a^{\mathrm{N}}=1.43 \mathrm{mT}, a^{\mathrm{H}_{1}}=1.15 \mathrm{mT}, a^{\mathrm{H}_{2}}=0.13 \mathrm{mT}$ [9], shown in Fig. 1. In the presence of superoxide dismutase (SOD) a drastic reduction in the DMPO-OOH signal was observed. This is thought to be a result of $\mathrm{O}_{2}^{--}$converting to water and oxygen before significant

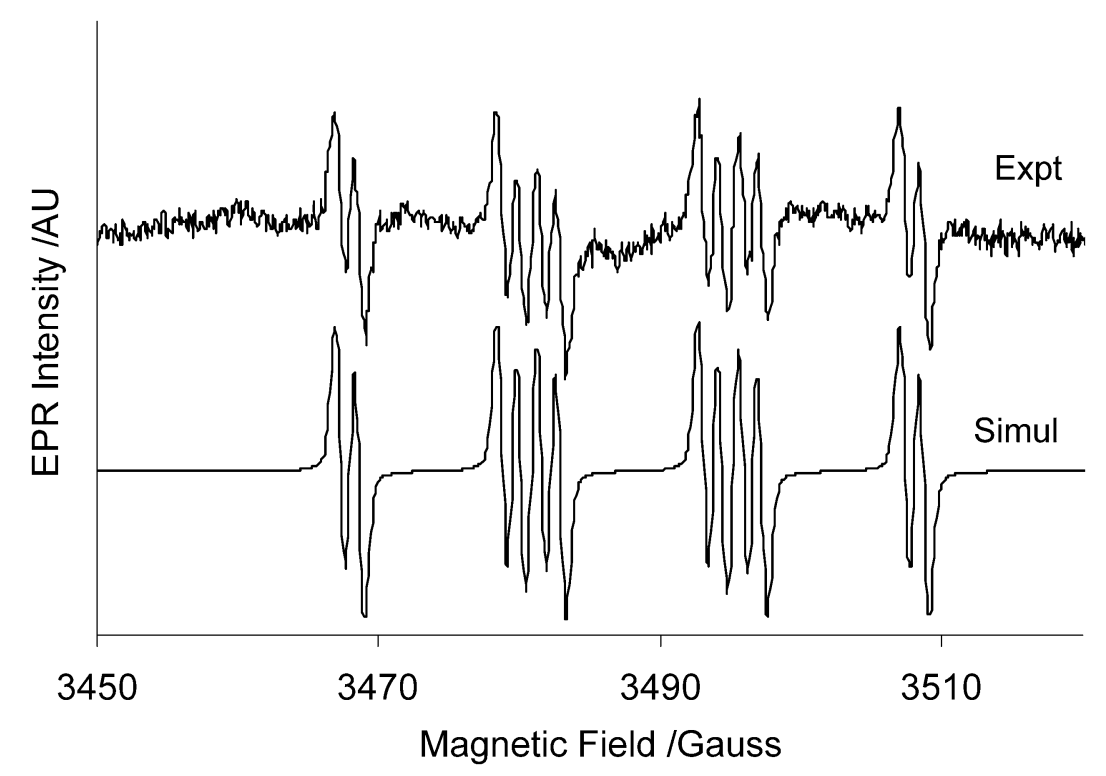

Figure 1. EPR spectrum showing 12 lines assigned to DMPO-OOH adduct obtained form xanthine/xanthine oxidase/DMPO in oxygenated medium. 
trapping occurs. Though the rate of spin trapping of superoxide radical is low, $10 \mathrm{M}^{-1} \mathrm{~s}^{-1}$ [13] compared to dismutation of $2.3 \times 10^{9} \mathrm{~m}^{-1} \mathrm{~s}^{-1}$ [16], large amounts of the spin trap would cause trapping to supersede dismutation in the elimination of the superoxide radical [14]. Diminution of $\mathrm{O}_{2}^{--}$caused a reduction in intensity of the DMPO-OOH spectrum. Thus SOD offers immense protection against damage by reactive oxygen species (ROS). Although $\mathrm{O}_{2}^{--}$and ${ }^{\circ} \mathrm{OH}$ are the two ROS implicated as most deleterious products of oxidative bursts in aerobic systems, the primary event leading to their formation has been much overlooked. This primary event must be the release of excessive amounts of electrons during metabolic processes. In the case of xanthine/xanthine oxidase, the substrate is oxidized to uric acid with release of electrons. Such free electrons would rapidly reduce available dioxygen to $\mathrm{O}_{2}^{--}$in aerobic systems, in the strict sense. Accumulation of the superoxide radical, an indicator of oxidative stress, initiates the series of ROS-associated reactions including formation of ${ }^{\circ} \mathrm{OH}$, the most damaging of oxygen derived radicals.

Replacement of DMPO in the xanthine with xanthine oxidase reaction by tiron gave EPR features showing the 4-line spectrum of a tiron-derived radical both in oxy- and deoxy-solutions (Fig. 2) presumably for the tiron- $\mathrm{O}_{2}$ adduct as expected of a spin trapping reaction. The four-line spectrum, however, is similar to that ascribed to the tiron-derived semiquinone [3]. This is a radical specie in which there are two hydrogens with different spin densities. Computer simulation gives two non-equivalent hydrogens with hyperfine couplings of 0.379 and $0.181 \mathrm{mT}$ assigned to hydrogens in ring positions 2 and 6 , respectively. By virtue of its $\beta$-position to the ring, the $\mathrm{OH}$ hydrogen is effectively shielded by oxygen and therefore gives negligibly little or no hyperfine coupling. Formation of the tiron semiquinone has been explained as shown in Eqs (1) and (2) above.

In an alternative procedure the experiment was performed in deoxygenated solution so that dioxygen was eliminated from the xanthine with xanthine oxidase system. Electrons released from xanthine in its oxidation to uric acid were, therefore, freely available to react with substrates other than oxygen. Tiron gave EPR features evident of formation of the semiquinone radical in the absence of $\mathrm{O}_{2}^{--}$as oxidant precursor, Fig. 2(b). This observation is of great importance in view of the notion that semiquinone iss formed by the direct oxidation of its parent compound. It is here proven that tiron is capable of reacting with hydrated electrons to form the observed radical.

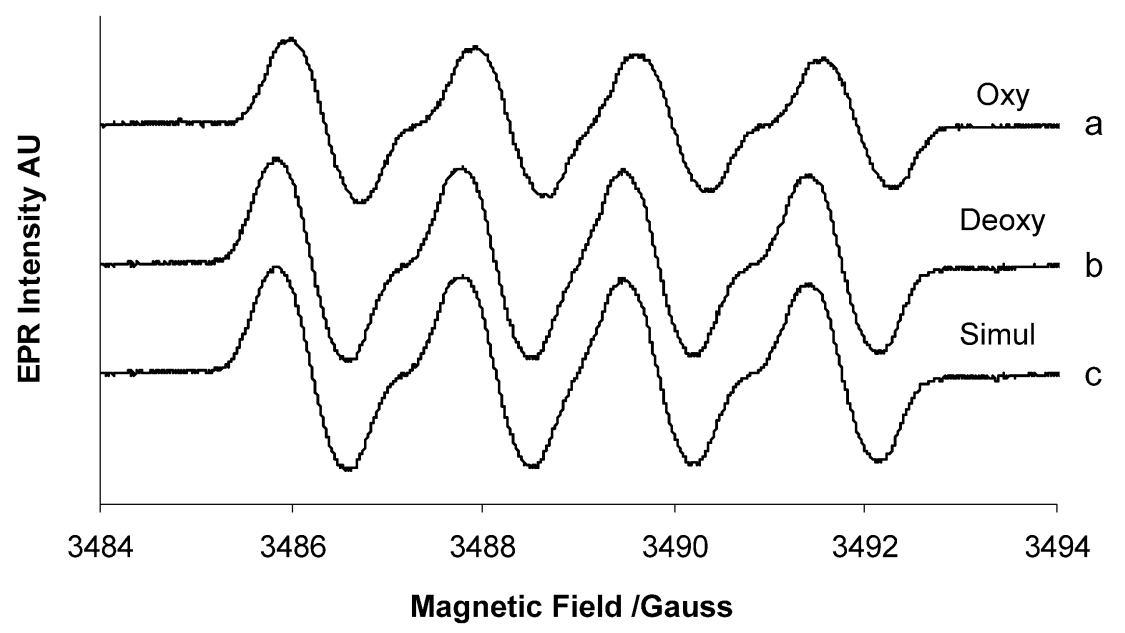

Figure 2. EPR spectra of tiron semiquinone obtained from xanthine/xanthine oxidase/tiron in (a) oxygenated, (b) deoxygenated media and (c) a simulation. 


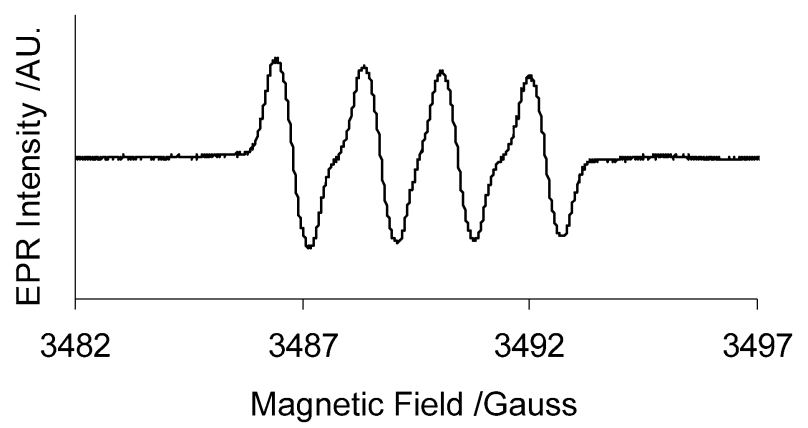

Figure 3. EPR spectrum of tiron semiquinone obtained from xanthine/xanthine-oxidase/tiron/DMPO.

Comparison of the spectra of tiron semiquinone in the presence and absence of oxygen shows that the line width is broader in the presence of oxygen $(0.0778 \mathrm{mT})$ than in deoxygenated solutions $(0.0733 \mathrm{mT})$. This is due to spin exchange by dissolved oxygen causing line-broadening effects in the EPR spectra [15].

Spectral features for tiron are similar both in oxygenated and deoxygenated conditions, indicating formation of tiron semiquinone irrespective of oxygen concentration. The spectrum under anoxic condition, however, is less intense and shows a rapidly decaying signal. Two possibilities can be considered; the direct reaction of tiron with hydrated electron or alternatively, the oxidation of tiron by a first-formed superoxide radical anion. Should the latter be case, DMPO-OOH would predominate in a system in which both tiron and DMPO were included. This was not the case as seen from the exclusive formation of tiron semiquinone, Fig. 3. In fact no DMPO-OOH was observed in competitive formation. This suggests reactivity of tiron with hydrated electrons to form the semiquinone detected both in oxy- and deoxyconditions. A competitive formation of the three possible radicals from oxygen, DMPO, and tiron would have shown a composite spectrum including also the DMPO-OOH with its characteristic 12-line spectrum. Observation of the tiron radical to the exclusion of all others must mean its exceptionally high rate of formation.

A close inspection of the spectra in Fig. 2 shows a steady loss in intensity of the EPR lines as the Hall field increases. This is characteristic of rapidly decaying species and explains the difference between experimental and simulated spectra. The decay rate must be significantly higher than the instrumental scan rate as set for the spectrum, hence the decreasing intensity of lines which are components of the same spectrum. This observation led us to do a kinetic scan during which the Hall field was set at the field position of the second of the four lines while the sweep width was set at zero so that a variation in intensity of the line was recorded in real time. Figure 4 shows a first-order kinetic decay profile obtained from a log plot of intensity vs. time (inset in Fig. 4) which is linear. A first-order rate constant of $1.8 \times 10^{-3} \mathrm{~s}^{-1}$ is derived from the plot.

A unique demonstration of direct formation of the semiquinone from tiron was performed by the electrolysis of tiron with in-situ detection by simultaneous EPR spectroscopy. Spectrum obtained, Fig. 5, shows the same 4-line spectrum, but all emerging with equal intensity. This spectrum accords with the simulation spectrum in which non-decaying species is a formal assumption. The electrolysis spectrum arises from a continuous generation of the tiron radical in a process that ensures steady state concentration of radicals in the EPR flat cell, hence a constant intensity of lines across the field axis. In the enzymatic system the release of electrons terminates after all the substrate hypoxanthine is converted to uric acid in a medium of excess oxygen or all available oxygen has been reduced, at which point 


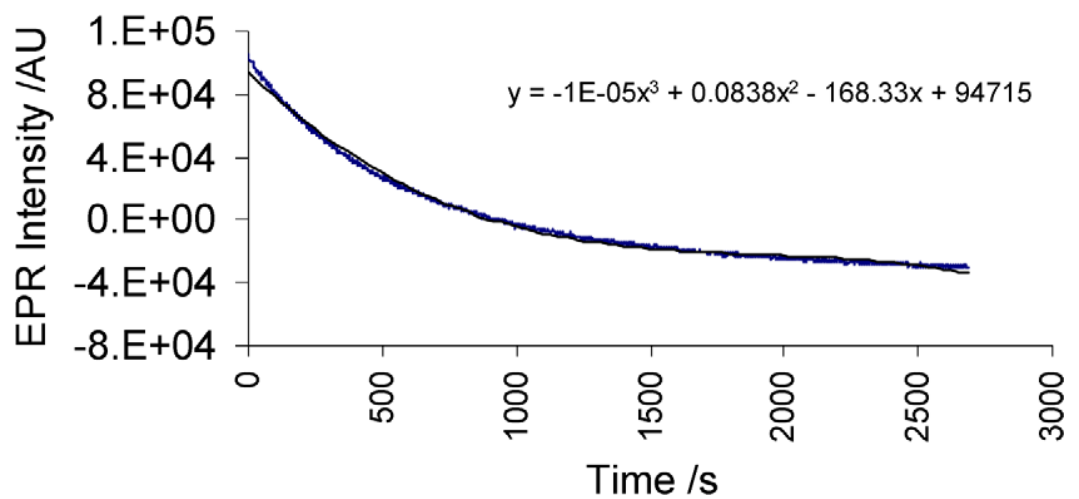

(a)

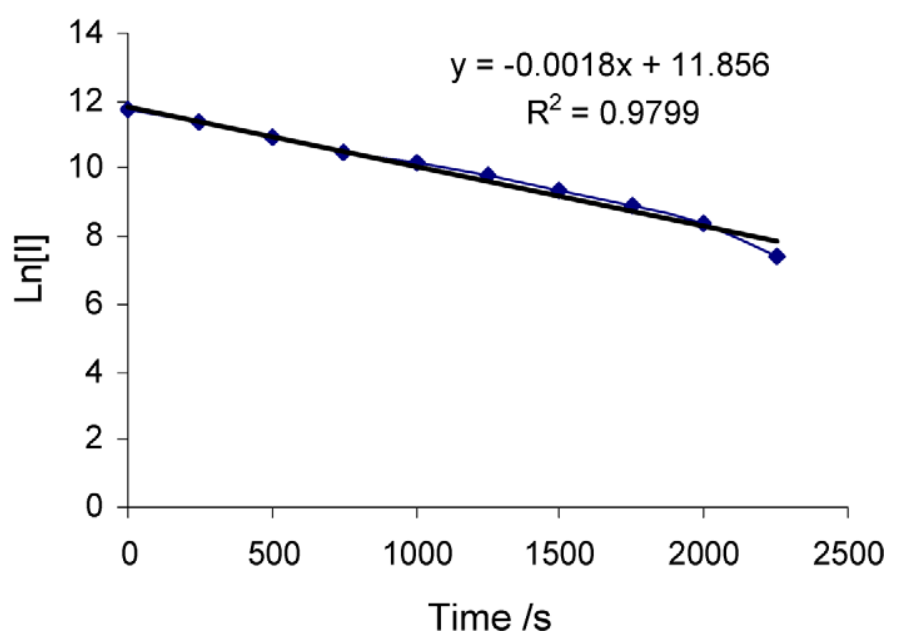

(b)

Figure 4. Kinetic EPR scan showing (a) decay of tiron semiquinone radical and (b) a log plot vs. time showing first-order kinetics of the decay.

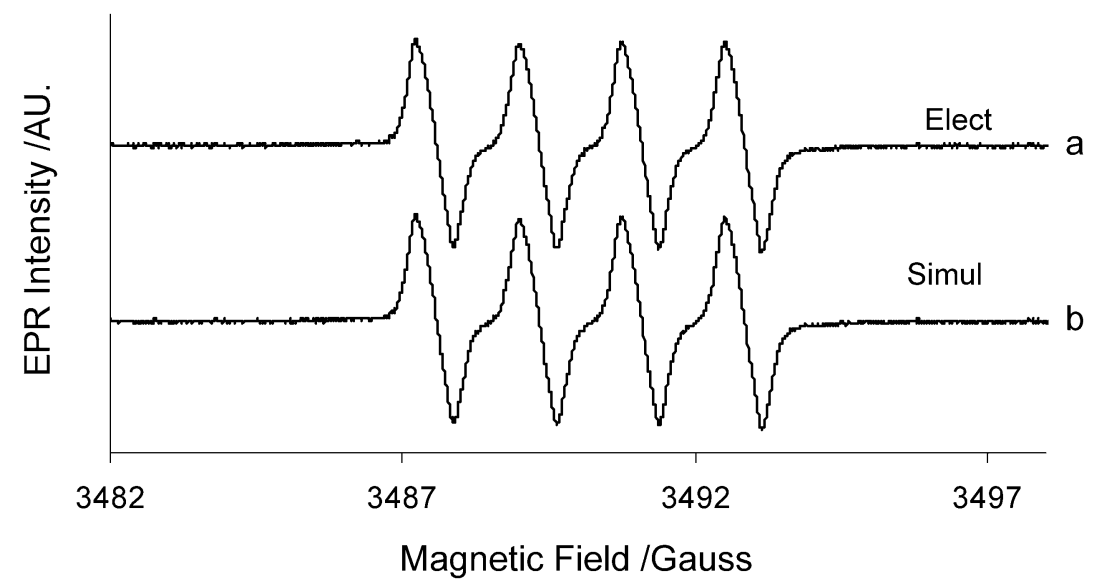

Figure 5. EPR spectrum obtained by (a) electrolysis of tiron in situ and (b) computer simulation. 
<smiles>O=S(=O)(O)c1cc(O)c(OCOc2c(O)cc(S(=O)(=O)O)cc2S(=O)(=O)O)c(S(=O)(=O)O)c1</smiles>

Scheme 2.

further generation of radicals will terminate and the observable concentration of radical begins to decay. Our experiment was set up with continual exposure to ambient oxygen so it is less likely that oxygen was depleted. Though a substrate (hypoxanthine) dependent variation was not done, this is thought to be more likely the case.

Contrary to the notion that tiron functions as a spin trap during mitigation of reactions mediated by reactive oxygen species, this is clearly not the case as tiron does not 'add on' the radical to form a new molecular adduct as is the case with nitroxide and nitroso spin traps. More precisely it is an electron trap. Our results show that tiron must be either an electron trap as it would react with electrons and the superoxide radical anion to form its semiquinone radical. This is clearly demonstrated by the direct trapping of electrons in the system comprising equimolar amounts of DMPO and tiron in the xanthine with xanthine oxidase reaction in which the tiron-derived radical was formed to the exclusion of DMPO$\mathrm{O}_{2} \mathrm{H}$. This also indicates that tiron is a much more efficient electron trap than $\mathrm{O}_{2}$. Were the contrary to be the case DMPO-OOH spectrum would be predominate or be seen in an initial composite spectrum before decaying as tiron semiquinone grows in, as in Eq. (1). Comparable efficiencies of electron capture by tiron and dioxygen would result in equilibrium between DMPO-OOH and the semiquinone radical both of which would have been recorded in the initial composite EPR spectrum.

On the stability of the superoxide radical anion, it may react as a powerful reducing species being able to lose an electron to revert to oxygen, or as an oxidising species by gaining a second electron thus forming the peroxide radical anion which rapidly protonates to form the hydroperoxide radical anion $\left(\mathrm{HOO}^{-}\right)$[9]. The fact that no DMPO-OOH was detected in the joint presence of DMPO and tiron in oxygenated xanthine oxidase/hypoxanthine system does not allow inference to be made on the behaviour of $\mathrm{O}_{2}^{--}$in solution with the tiron semiquinone. Had any $\mathrm{O}_{2}^{--}$been formed, it was either too little to be detected or had rapidly reacted with tiron, the free radical of which predominates. It is curious to observe that whereas there is decay in the oxy-spectrum in Fig. 2 as expected for depleting hypoxanthine in oxic solution, the deoxy spectrum also shows a decay profile. This we think must be due to recombination of first formed tiron radicals. In the absence of oxygen, there is a possibility of radical-radical recombination to form a dimer; as illustrated in Scheme 2.

\section{Acknowledgement}

The author is grateful to De Montfort University, Leicester, United Kingdom, for research support.

\section{References}

[1] C.M. Krishna, J.E. Liebmann, D. Kaufman, W. DeGraff, S.M. Hahn, T. McMurry, J.B. Mitchell and A. Russo, The catecholic metal sequestering agent 1,2-dihydroxybenzene-3,5-disulfonate confers protection against oxidative cell damage, Arch. Biochem. Biophys. 294 (1992), 98-106. 
[2] B. Poeggeler, S. Thuermann, A. Dose, M. Schoenke, S. Burkhardt and R. Hardeland, Melatonin's unique radical scavenging properties - roles of its functional substituents as revealed by a comparison with its structural analogs, J. Pineal Res. 33 (2002), 20-30.

[3] A.N. Ledenev, A.A. Konstantinov, E. Popova and E.K. Ruuge, A simple assay of the superoxide generation rate with tiron as an EPR-visible radical scavenger, Biochem. Int. 13(2) (1986), 391-396.

[4] G. Van Ginkel and J.K. Raison, Light induced formation of oxygen radicals in systems containing chlorophyll, Photochem. Photobiol. 32 (1980), 793.

[5] V. Kahn and R.W. Miller, Tiron as a substrate for mushroom tyrosinase, Phytochemistry 26(9) (1987), 2459-2466.

[6] L.L. Herscher, M.C. Krishna, J.A. Cook, C.N. Coleman, J.E. Biaglow, S.W. Turtle, F.J. Gonzalez and J.B. Mitchell, Protection against SR4233 (Tirapazamine) aerobic cytotoxicity by the metal chelators desferrioxamine and tiron, Int. J. Radiat. Oncol. Biol. Phys. 30(4) (1994), 879-885.

[7] T. Kiss, I. Sóvágó and R.B. Martin, Complexes of 3,4-dihydroxyphenyl derivates. $\mathrm{Al}^{3+}$ binding to catecholamines and tiron, J. Am. Chem. Soc. 111(10) (1989), 3611-3614.

[8] A.V. Peskin, Y.A. Labas and A.N. Tikhonov, Superoxide radical production by sponges Sycan sp., FEBS Lett. 434(1/2) (1998), 201-204.

[9] L.H. Patterson and F.A. Taiwo, Electron paramagnetic spectrometry evidence for bioreduction of tirapazamine to oxidising free radicals under anaerobic conditions, Biochem. Pharmacol. 60 (2000), 1933-1935.

[10] Q.-S. Qui, A.-G. Liang, H.-J. Zheng and P. Chen, $\mathrm{Ca}^{2+}$ calmodulin-stimulated superoxide generation by purified plasma membrane from wheat roots, Plant Science 101(2) (1994), 99-104.

[11] F.A. Taiwo, H.J. Powers, E. Nakano, H.R. Griffiths and D.F. Nugent, Free radical reactions in atherosclerosis; An EPR spectrometry study, Spectroscopy 20 (2006), 67-80.

[12] F.A. Taiwo, P.M. Brophy, D.I. Pritchard, A. Brown, A. Wardlaw and L.H. Patterson, Cu/Zn superoxide dismutase in excretory-secretory products of the human hookworm Necator americanus. An electron paramagnetic spectrometry study, Eur. J. Biochem. 264(2) (1999), 434-438.

[13] G.M. Rosen and E.J. Rauckman, Spin trapping of free radicals during hepatic microsomal lipid peroxidation, PNAS (USA) 78(12) (1981), 7346-7349.

[14] R. Lauricella, A. Allouch, V. Roubaud, J.-C. Bouteiller and B. Tuccio, A new kinetic approach to the evaluation of rate constants for the spin trapping of superoxide/hydroperoxyl radical by nitrones in aqueous media, Org. Biomol. Chem. 2 (2004), 1304-1309.

[15] H.M. Swartz and R.B. Clarkson, The measurement of oxygen in vivo using EPR techniques, Phys. Mod. Biol. 43 (1999), 1957-1975.

[16] E. Finkelstein, G.M. Rosen and E.J. Raukman, Spin trapping kinetics of superoxide and hydroxyl radicals with nitrones, J. Am. Chem. Soc. 102 (1980), 4994-4996.

[17] R.F. Anderson, M.A. Brimble, M.R. Nairn and J.E. Packer, A comparison of the one electron reduction of kalafungin and analogues with other semiquinones using pulse radiolysis, J. Chem. Soc., Perkin Trans. 2 (1999), 475-479. 


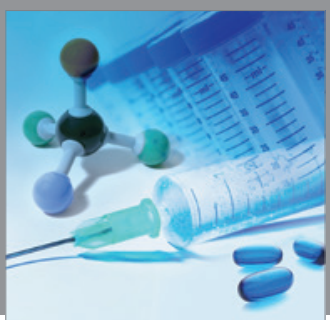

International Journal of

Medicinal Chemistry

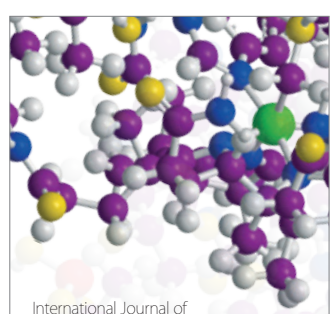

Carbohydrate Chemistry

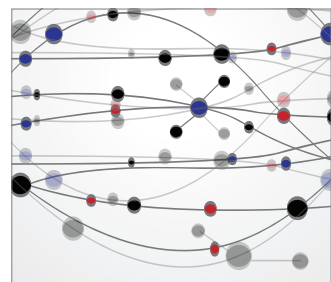

The Scientific World Journal
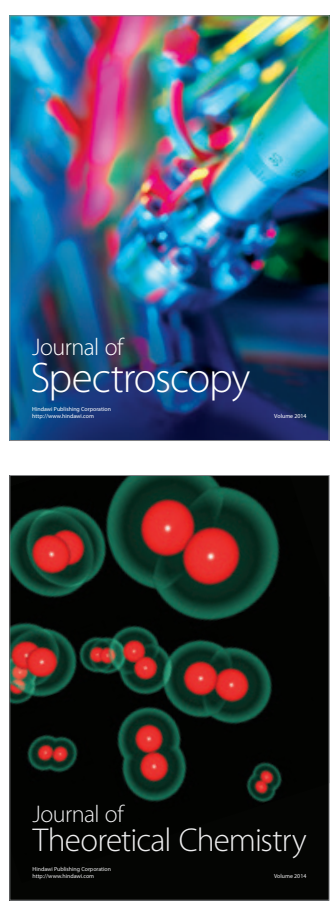
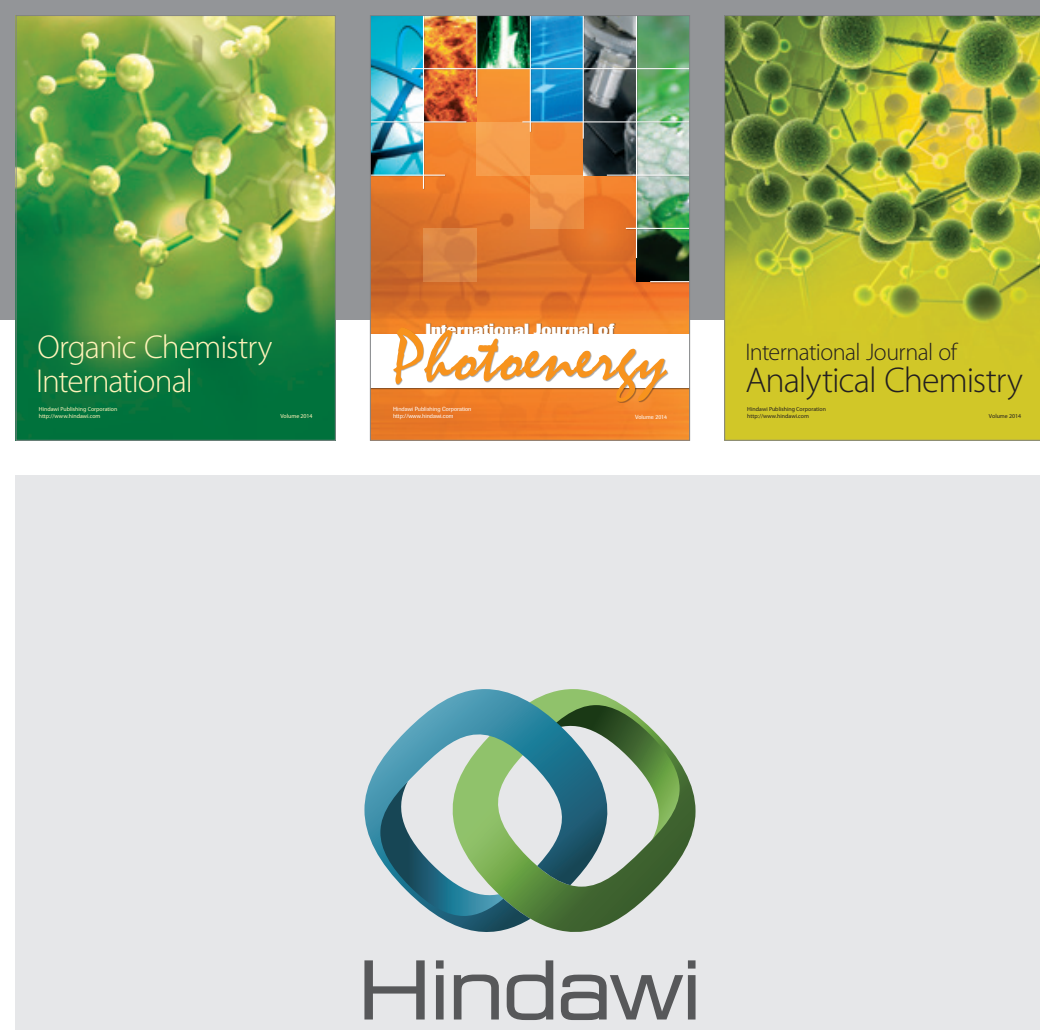

Submit your manuscripts at

http://www.hindawi.com
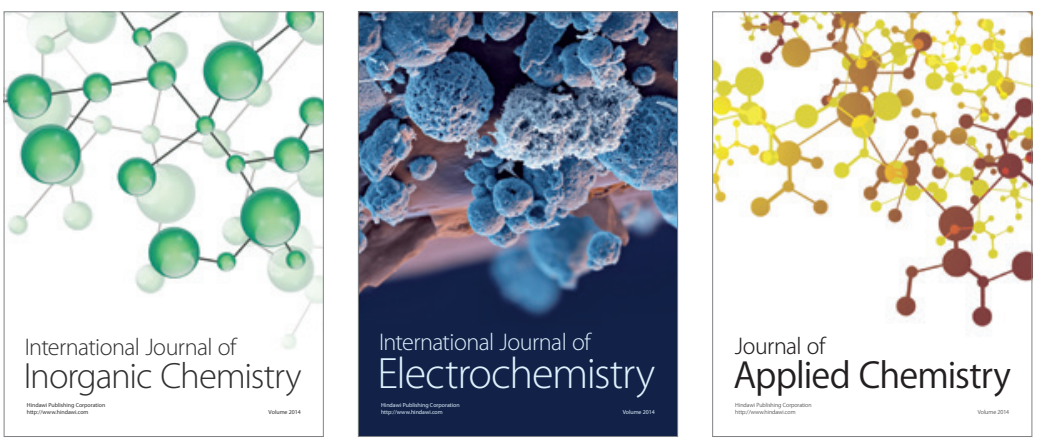

Journal of

Applied Chemistry
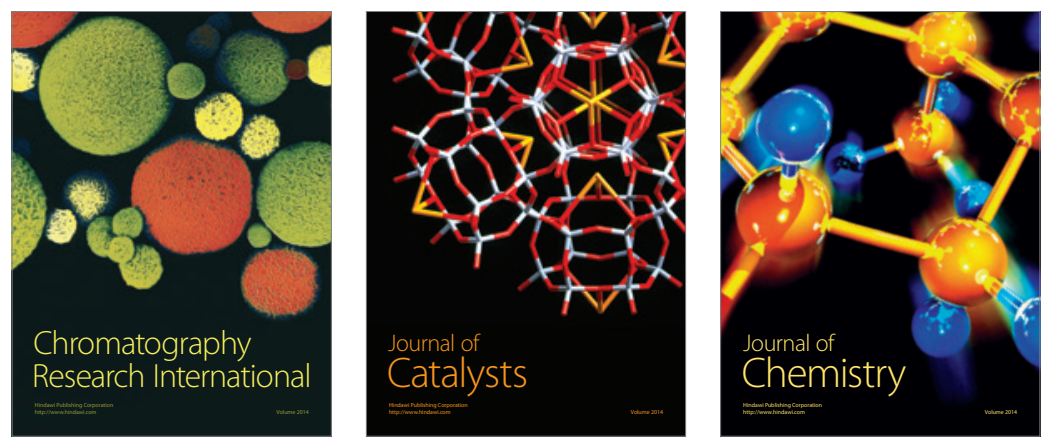
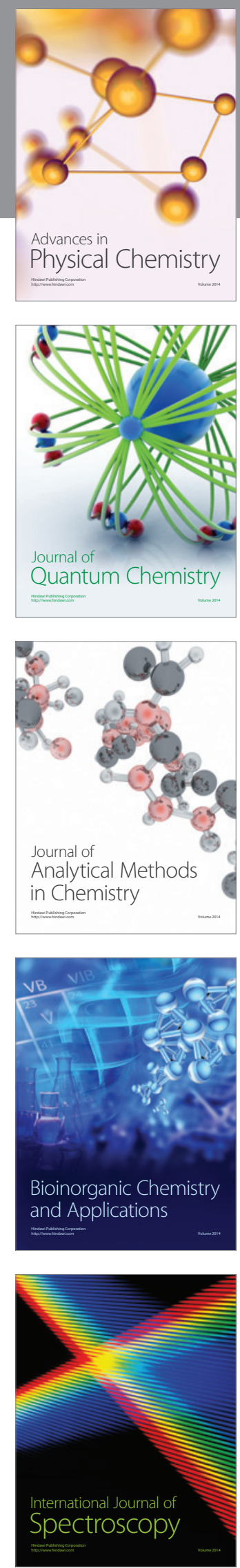